\title{
Non-invasive Functional Brain Biomarkers for Cognitive-Motor Performance Assessment: Towards New Brain Monitoring Applications
}

\author{
Rodolphe J. Gentili ${ }^{1,2}$ \\ ${ }^{1}$ Cognitive Motor Neuroscience Laboratory \\ Department of Kinesiology \\ School of Public Health University of Maryland \\ College Park, MD 20742, USA \\ ${ }^{2}$ Neuroscience and Cognitive Science Program University of Maryland \\ College Park, MD 20742, USA \\ rodolphe@umd. edu
}

\begin{abstract}
Along with theoretical advances in neuroscience research, recent neurotechnological developments provide portable recording and processing systems that can be employed for real-time assessment in applied military environments. This article provides a brief overview of research related to noninvasive brain biomarkers derived from brain signals that can track brain dynamics during cognitive-motor performance. Potential applications of such brain biomarkers for military personnel such as neurofeedback for accelerated learning as well as brain monitoring for performance assessment and rehabilitation are discussed.
\end{abstract}

Keywords: Cognitive-motor performance, EEG/fNIRS biomarkers, alpha power, phase synchronization, brain monitoring, neurofeedback, rehabilitation.

\section{Introduction}

In parallel with the continuous advancements of neuroscience, the recent developments of neurotechnology are able to provide wearable sensors and portable recording systems that can be employed for real-time assessment in applied environments [1],[2]. Thus, the idea to transfer some current techniques from laboratories to the field is increasingly being considered. Among the various avenues of applied neurosciences, applications in clinical settings and in the operational realm could be highly beneficial to the military as well as commercial/industrial communities [2]. In particular, when considering the military environment, it is widely accepted that "the Soldier is the acknowledged centerpiece of the Army's warfighting system, and success largely depends on the mental status of these individuals." ([3], p.1). Therefore, there is a need to develop rigorous neuropsychological/neurological assessment techniques, which would represent a major advance in Soldier performance research [3],[4]. Specifically, the training of individuals, as well as the monitoring of individual cognitive-motor states during performance, appear to be two important avenues that need to be considered in operational settings [2]. 
Training and monitoring of cognitive-motor performance can be based on the combination of behavioral analyses as well as on the investigation of brain dynamics. Brain activity assessment can provide sensitive indicators to evaluate the brain/mental status of the performer. For instance, while behavioral outcomes are informative on the quality of performance, the performance can be influenced by multiple confounded factors (e.g., fatigue, workload, level of expertise, etc.), which could be revealed by accessing brain/mental status.

Such assessment is possible by employing non-invasive embedded brain monitoring systems based on specific indicators or brain biomarkers to track brain dynamics. Additionally, such monitoring tools should allow for the tracking of brain dynamics in ecological situations in which humans learn new tasks, master novel tools, and/or adapt to changing environments. Thus, these brain biomarkers should not only be non-invasive (i.e., no surgical intervention needed), but also simple to record and analyze in addition to being robust and sensitive to specific changes in brain function in natural situations. Electroencephalography (EEG) is well suited for such assessment in situations that require non-invasive recording of the dynamic brain activity with high temporal resolution (e.g., millisecond). In addition, hemodynamic signals such as functional near infra-red spectroscopy (fNIRS) can also be recorded by portable devices and provide a good complementary method to EEG [5]. Over the years, multiple research efforts (e.g., [6],[7],[8],[9],[10],[11],[12],[13],[14],[15],[16]) proposed methods and provided such biomarkers that could be employed to track the brain status during cognitive-motor performance.

This article, will first overview elements related to brain biomarkers of cognitivemotor performance. Then, potential applications in a military context such as neurofeedback for accelerated learning as well as brain monitoring for veteran rehabilitation and performance assessment will be discussed.

\section{Elements of Overview}

This section will focus mainly on brain biomarkers associated with cognitive-motor performance derived from EEG and to a lesser extent from fNIRS. As mentioned above, both can be recorded by portable systems and, therefore, are particularly well suited for brain assessment during cognitive-motor performance (e.g., aiming, reaching) in the field.

\subsection{Spectral Power}

Since the seminal work conducted by Hatfield and colleagues [10] close to three decades ago, a growing body of evidence suggests that it is possible to assess the cortical dynamics of cognitive-motor skills in expert performers during visuomotor tasks. These investigations revealed progressive changes in EEG during skill learning and also differences in the level of EEG power between novice and expert sport performers [8],[9],[11],[14],[13],[14],[15],[16]). Particularly, alpha ( 8-13 Hz) and theta $(\sim 4-7 \mathrm{~Hz})$ power were positively related to the level of cognitive-motor performance [8],[11],[12],[13]. For the sake of clarity and conciseness, only the results related to alpha power are presented here. However, theta oscillations also 
appear to be related to performance enhancement and, specifically, an increasing body of evidence supports that theta oscillations could be functionally related to error monitoring [9],[17], [18].

Namely, previous investigations reported that experts demonstrated an overall increase in EEG alpha power compared to novices in the left temporal region during a precision aiming task [11],[12]. The results suggest that differences in EEG alpha power are related to the differences in the level of mastery of the cognitive-motor task. In general, such EEG changes are indicative of high levels of skill and associated with a cortical refinement leading to reductions of nonessential cortical resources to perform the task [11]. This is consistent with the idea that a high level of alpha power corresponds to a reduced activation of a given cortical region indicating a reduction of the recruitment of neural resources [11]. Also, these differences in cortical dynamics between novices and experts mirror important performances discrepancies. Namely, the experts scored higher and exhibited lower performance variability compared to the novices. Thus, these studies can contribute to the development of brain biomarkers (e.g., changes in EEG alpha power) capable of identifying a high level of cognitivemotor performance resulting from an extensive practice period. However, this research did not focus on the evolution of such brain biomarker throughout the training period itself.
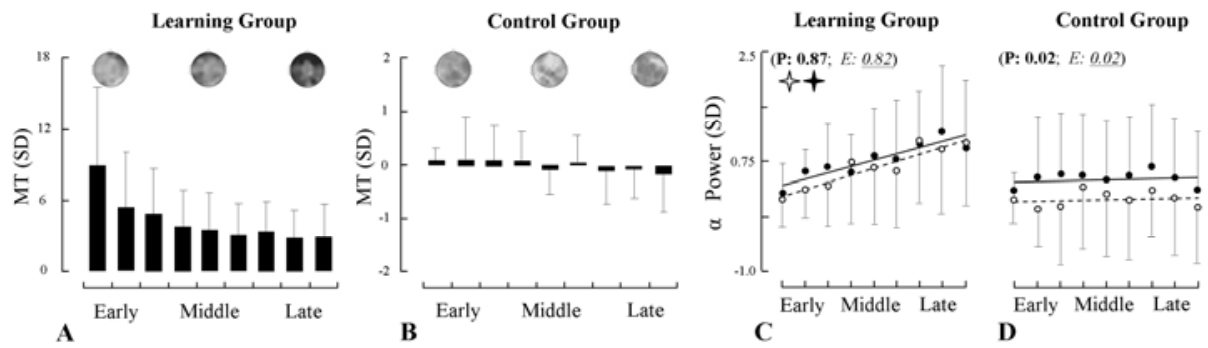

Fig. 1. Concomitant EEG and kinematic changes throughout adaptation for the learning and control groups. Changes in movement time (MT) for the learning (A) and the control (B) groups for early, middle, and late adaptation. The scalp plots represent the changes in alpha power through the same practice period for the planning stage. Changes in the magnitude of the standardized (sd units) EEG alpha power during planning (black circles) and execution (empty circles) for the learning (C) and control (D) groups. (Adapted from [9]).

Cortical changes during learning were examined in a recent investigation that assessed changes in brain dynamics throughout a marksmanship intensive training of novice performers from the US Naval Academy during three months [13]. The findings revealed that throughout the training period the shooting performance was enhanced along with an increase in alpha power at the (contralateral) left temporal site while such a result was not observed when participants were at rest. Recently, this research has been extended by employing movements related to daily activities (e.g., reaching, grasping; [9],[16]). This research employed a shorter learning timeframe (hours instead of weeks/years) of a new cognitive-motor skill and assessed interferences with previously acquired motor experiences [9]. This latter aspect is 
important since, in practice, Soldiers often need to switch/adapt rapidly from one task to another, potentially inducing interference between tasks. In this study, EEG brain signals were analyzed from participants divided into two groups: i) a learning group that had to learn a new visuomotor transformation while performing drawing movements requiring suppression of familiar cognitive-motor responses, and ii) a control group for whom no visual transformation had to be learned [9]. The findings revealed that as participants of the learning group enhanced their cognitive-motor performance, EEG frontal alpha power during both movement planning and execution was progressively increased. It was suggested that such changes reflected initial involvement of frontal executive functioning to suppress established visuomotor mappings followed by a progressive idling [9]. No change in EEG alpha power nor performance was observed in the control group.

Thus, altogether this research suggests that changes in EEG alpha power can be used as non-invasive functional brain biomarkers either to assess the level of mastery of a particular cognitive-motor skill and/or to track the evolution of brain dynamics throughout cognitive-motor training.

\subsection{Phase Synchronization}

Besides biomarkers derived from EEG power, phase synchronisation (coherence, phase locking value (PLV)) of EEG signals that reflect the degree of cortico-cortical communication also provide brain biomarkers for cognitive-motor performance.

Although relatively less common than studies investigating EEG power, several recent investigations focusing on the degree of cortico-cortical communications in relation to changes in cognitive-motor performance have been conducted [6],[7],[16],[19]. Generally, these studies have provided convergent evidence that skilled individual exhibited a reduced level of coherence between cortical areas. For instance, EEG coherence between frontal and several other cortical regions in two groups of highly skilled marksmen who were similar in expertise, but who differed in competitive performance history were compared. One group performed consistently better in competition and exhibited significantly lower coherence between the left temporal region and the premotor area in the high-alpha $(10-13 \mathrm{~Hz})$ and low-beta (13-22 Hz) bandwidths during the aiming period [6]. Similarly, it was shown that coherence could assess brain dynamics in relation to the level of mastery of a motor task. Particularly, expert marksmen exhibited lower coherence over the whole scalp compared to novices, with the effect most prominent in the right hemisphere. Coherence was positively related to aiming movement variability in experts [7]. A reduction in coherence is generally interpreted as a refinement of cortical networks in experts reflecting a reduction of nonessential functional communications among the cortical regions of interest associated to a superior performance [11].

Recently, (similar to the EEG power, see section 2.1) this research has been extended by considering a short period of training during which participants had to learn a new visuomotor task while inhibiting prepotent cognitive-motor responses [20]. The findings revealed a decrease of phase synchronization (PLV) for both movement planning and execution as participants adapted throughout training. These changes were correlated with enhanced kinematics as the task progressed. 

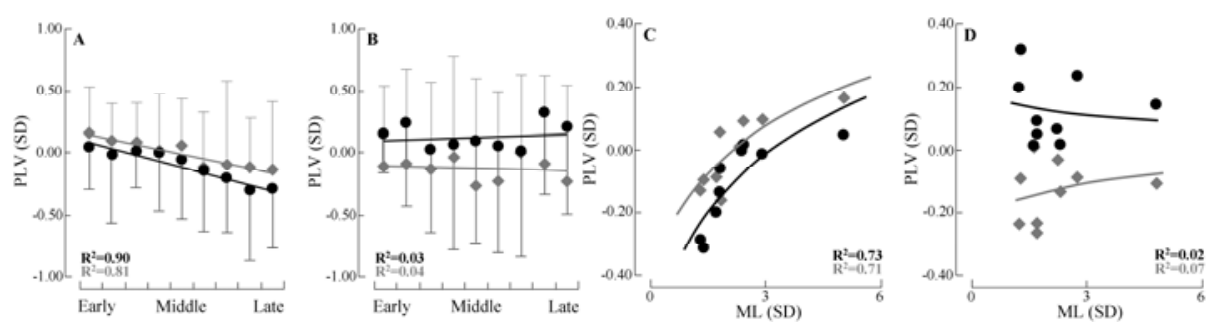

Fig. 2. Changes in PLV for the learning (A) and control (B) groups throughout practice during the planning stage. PLV versus movement length (ML) for the learning (C) and the control (D) groups. Standard deviation (SD) is used as unit. The black and gray color represent the PLV values for the pair of electrodes Fz-F4 (high beta band) and Fz-C4 (gamma band), respectively.

Therefore, as a whole, changes in EEG coherence can be used as additional functional brain biomarkers to assess the level of a particular cognitive-motor skill and/or to track brain dynamics during cognitive-motor training.

\subsection{Functional Near Infra Red Spectroscopy and Hemodynamic Responses}

Besides EEG it is also important to investigate hemodynamic changes by using alternative approaches such as fNIRS to derive brain biomarkers related to cognitivemotor performance. While EEG measures electrical activity, fNIRS measures blood oxygenation levels in the brain, providing a different, possibly complementary, source of information about brain functioning that is accessible with portable recording systems [1],[5]. fNIRS is an emerging optical brain imaging modality that measures hemodynamic response in order to provide biologically relevant indicators for brain functions such as cognitive workload [21] and cognitive-motor performance [22],[23]. These qualities make fNIRS well-suited for assessing brain status during ecological situation in the field [21],[22]. Specifically, several studies investigated the brain activity by employing fNIRS when comparing cognitive-motor skills in novice and expert performers during training [22],[23]. For instance, by analyzing fNIRS signals recorded in prefrontal regions and manual dexterity, technical skill of expert and novice surgeons while performing a surgical knot-tying task were compared [22]. The findings revealed a decrease in relative changes of total and oxygenated hemoglobin as well as an increase in deoxygenated hemoglobin throughout training. It was concluded that learning-related refinements in performance were mediated by reductions in prefrontal activation. Recently, by using a task where participants had to learn a novel visuomotor transformation while suppressing familiar motor plan (see section 2.1), the changes in fNIRS recorded at the prefrontal regions along with changes in performance were investigated [23]. Preliminary results revealed that throughout training the performance was enhanced along with a simultaneous and progressive reduction in prefrontal oxygenated hemoglobin.

The high prefrontal activation during early learning may reflect a primary role of the inhibitory processes to suppress familiar motor responses of inappropriate actions while such a role is reduced during late learning reflected by a smaller prefrontal activation [23]. It must be noted that the enhancement of the performance along with a reduction of the prefrontal activity are in accordance with results revealing an increase in prefrontal alpha power previously reported with the same task [9]. 

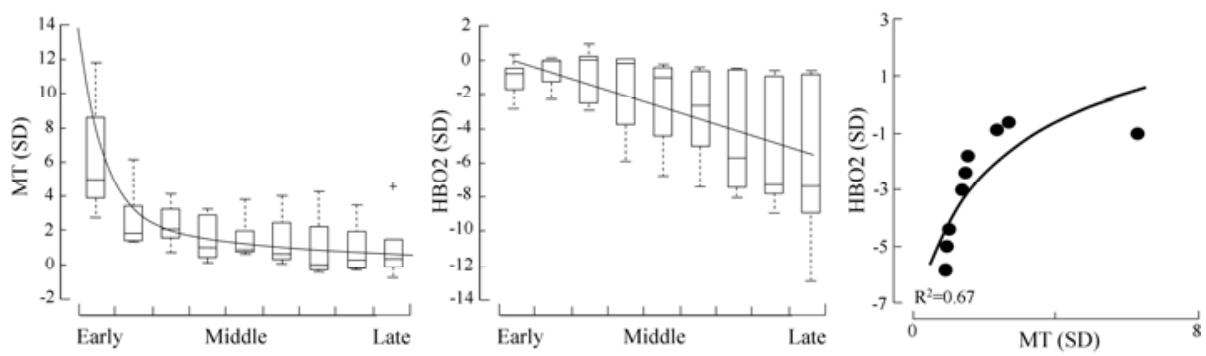

Fig. 3. Changes in movement time (MT; left panel); in oxygenated hemoglobin (HBO2; middle panel) throughout adaptation for the learning group. Oxygenated hemoglobin versus movement time (MT) for the learning groups (right panel). Standard deviation (SD) is used as unit (Adapted from [23]).

Therefore, although additional research is needed, changes in oxygenation, deoxygenation, total haemoglobin extracted from fNIRS signals seem to be promising to provide (hemodynamic-based) non-invasive functional brain biomarkers to assess the level of a particular cognitive-motor skill and/or to track the evolution of brain status during cognitive-motor practice.

\section{Future Potential Applications}

\subsection{Neurofeedback and Accelerated Learning}

A possible area of application of these EEG/fNIRS biomarkers would be the training of military personnel. "Today's warfighter is required to master a large number of diverse skills spanning the range of cognitive and motor domains in increasingly rapid timeframes" [24]. Thus, the training/learning of cognitive-motor performance is often performed under time pressure and may require the acquisition of several tasks at the same time. Thus, there is a growing interest to develop methods to enhance/accelerate the learning.

A possible method to enhance/accelerate learning would be to use augmented feedback with neurofeedback (or biofeedback) systems. Neurofeedback (or biofeedback) is a training technique that measures processes and provides feedbacks (e.g., visual, auditory) based on the neural activity (e.g., EEG) of individuals to help these individuals learn to control/change their brain activity. Although, further research seems to be needed, one possible application of neurofeedback with healthy individuals is enhancement of cognitive-motor performance [25]. For instance, based on previous results associating the changes in EEG alpha power at specific scalp sites and performance outcomes, it was shown that neurofeedback could significantly improve shooting performance in twenty four skilled archers [26]. The effect of EEG neurofeedback training was also examined in golfers, comparing performance enhancement with and without neurofeedback [27]. It was shown that the overall percentage of successful putts was significantly greater once neurofeedback was administered. Moreover, combined with additional findings (e.g., performance related 
to memory, attention, creativity and mood [28],[29]) it appears that neurofeedback can elicit positive changes in performance and thus has promising implications to enhance/accelerate cognitive-motor performance.

Therefore, since the functional biomarkers previously described were able to track changes in cerebral cortical dynamics during learning of a new cognitive-motor skill, it would seem reasonable to integrate them in a neurofeedback system to provide augmented feedback to individuals and thus analyze how this would enable enhanced/accelerated learning. Thus, this augmented (brain-based) feedback could be combined with feedbacks from the behavior that should allow the participants to learn faster/better compared to the situation when only 'classical' feedbacks are available [27]. When considering the augmented feedback, (without overloading the information processing capacity of individuals) multimodal augmented feedback could be considered including EEG (e.g., power, coherence) and fNIRS (e.g., oxygenated hemoglobin) biomarkers to provide a more robust, accurate and efficient feedback during the learning of new cognitive-motor skills. However, general (e.g., directionality of the changes in power and coherence) as well as specific (e.g., brain sites) features of these biomarkers depend on several factors (such as the nature of the task; task requiring inhibition of prepotent cognitive-motor responses or not, etc.) and thus should be taken into account in neurofeedback systems design.

\subsection{Brain Monitoring for Cognitive-Motor Performance Assessment}

\section{Military Personnel}

Another application of these brain biomarkers would be to monitor brain activity for cognitive-motor performance assessment in military personnel performing a specific task. An application that seems particularly well-suited would be to monitor brain activity when behavioral performance may be not directly accessible. This is typically the case of the remote warfighters where Soldiers are unseen from other team members, operating in remote outpost (e.g. inside building in urban conflict) [3]. Thus, the recording and processing of objective brain biomarkers for such individuals would allow brain monitoring related to performance, fatigue, workload or stress/emotional status assessment [3],[30]. Thus, biomarkers related to cognitivemotor performance could also be combined with others related to specific mental states such as fatigue [31], workload [32] or stress/emotional responses [33]. For instance, it has been recently suggested that changes in EEG coherence would provide a metric that could monitor workload/task-related mental demand and assess cognitive-motor learning [32]. Moreover, it was also suggested that the commonly observed high level of alpha power this is generally associated to the mastery of cognitive-motor skills in the left temporal region appears to be reduced when individuals perform under pressure [33]. In the same vein, theta power increase can be related to fatigue and reduced alertness [31] as well as performance enhancement [17],[9]. Interestingly, changes in theta power related to drowsiness are reflected by the low component $(\sim 4-5 \mathrm{~Hz})$ located at the central and posterior sites of the frontal regions [31],[34] while those observed for cognitive-motor performance enhancement are reflected by the high component $(\sim 6-7 \mathrm{~Hz})$ located at the anterior and lateral sites of the frontal regions [23]. Therefore, when combining these various biomarkers it would possible to disentangle and thus assess, to some extent, separately the level of 
cognitive-motor performance, stress, fatigue and workload. It is important to note that even if the behavioral performance is available, the monitoring of brain states is still important since a poor cognitive-motor performance can result from several factors (e.g., stress, workload, fatigue, etc.) that will affect the behavior. Thus, such brain status assessment could help to provide a more accurate and relevant decision making tool related to Soldier and task force management. For instance, if the level of fatigue or stress is becoming too high, the Soldier should be replaced before he/she makes any mistakes. In the future, brain monitoring systems based on multiple biomarkers (e.g., EEG power, coherence) could allow identifying when Soldiers are incapacitated and predict forthcoming human failure. By providing robust brain status assessments, such multimodal brain monitoring systems could be particularly significant as a part of a more general system that can automatically take over from the human in the loop in order to prevent any critical failure [3].

\section{Rehabilitation of Veterans}

Another interesting avenue for brain monitoring applications would be the rehabilitation of veterans and specifically to provide assessment tools for diagnostic, prediction and recovery. This seems particularly relevant for veterans having cognitive-motor problems (e.g., post-traumatic stress disorder, traumatic brain injury) such as the "Invisible Wounded Soldier" where the integrity of brain functions has been compromised. Thus, in addition to the information provided by the usual behavioral performance, brain biomarkers specifically engineered for a particular impairment could provide critical metrics for diagnosis (e.g., the Soldier is able to return to duty if desired, impairment identification, etc.). Similarly, the combination of behavior and brain biomarkers could be employed for cognitive-motor restoration by providing complementary information that would allow identifying potential cerebral compensation mechanisms. One promising direction would be to employ such biomarkers to assess the dynamics of the cognitive workload capacity recovery in veterans throughout the rehabilitation process. In such a context, heightened mental workload could be indicative of compromised brain processes resulting from cognitive impairments. In addition to the EEG power and coherence previously mentioned [32], metrics such as event related potentials (ERPs) could also be employed as reliable indices of mental workload [35].

Therefore, the combination of brain biomarkers from (e.g., EEG power, coherence, ERPs, and fNIRS) different brain imaging modalities could provide a higher degree of confidence in assessment tools that would enable efficient and robust brain monitoring systems to enhance cognitive-motor performance.

\section{References}

1. Parasuraman, R.: Neuroergonomics: research and practice. Theor. Issues Ergon. Sci. 4, 520 (2003)

2. Kruse, A.: Operational neuroscience: Neurophysiological measures in applied environments. Aviation, Space and Environmental Medicine 78(5), 191-194 (2007)

3. Friedl, K.E., Grate, S.J., Proctor, S.P., Ness, J.W., Lukey, B.J., Kane, R.L.: Army research needs for automated neuropsychological tests: monitoring soldier health and performance status. Arch. Clin. Neuropsychol. 22, 7-14 (2007) 
4. Letz, R.: Continuing challenges for computer-based neuropsychological tests. Neurotoxicology 24, 479-489 (2003)

5. Coyle, S.M., Ward, T.E., Markham, C.M.: Brain-computer interface using a simplified functional near-infrared spectroscopy system. J. Neural Eng. 4(3), 219-226 (2007)

6. Deeny, S.P., Haufler, A.J., Saffer, M., Hatfield, B.D.: Electroencephalographic coherence during visuomotor performance:a comparison of cortico-cortical communication in experts and novices. J. Mot. Behav. 41, 106-116 (2009)

7. Deeny, S.P., Hillman, C.H., Janelle, C.M., Hatfield, B.D.: Cortico-cortical communication and superior performance in skilled marksmen: An EEG coherence analysis. J. Sport and Exercise Psychology 25, 188-204 (2003)

8. Del Percio, C., Rossini, P.M., Marzano, N., Iacoboni, M., Infarinato, F., et al.: Is there a "neural efficiency" in athletes? A high-resolution EEG study. Neuroimage 42(4), 15441553 (2008)

9. Gentili, R.J., Bradberry, T.J., Oh, H., Hatfield, B.D., Contreras-Vidal, J.L.: Cerebral cortical dynamics during visuomotor transformation: Adaptation to a cognitive-motor executive challenge. Psychophysiology (in press)

10. Hatfield, B.D., Landers, D.M., Ray, W.J.: Cognitive processes during self-paced motor performance: an electroencephalographic profile of skilled marksmen. J. Sport Psychol. 6, 42-59 (1984)

11. Hatfield, B.D., Haufler, A.J., Hung, T.M., Spalding, T.W.: Electroencephalographic studies of skilled psychomotor performance. J. Clin. Neurophysiol. 21(3), 144-156 (2004)

12. Haufler, A.J., Spalding, T.W., Santa Maria, D.L., Hatfield, B.D.: Neurocognitive activity during a self-paced visuospatial task: comparative EEG profiles in marksmen and novice shooters. Biol. Psychol. 53(3), 131-160 (2000)

13. Kerick, S.E., Douglass, L.W., Hatfield, B.D.: Cerebral cortical adaptations associated with visuomotor practice. Med. Sci. Sports Exerc. 36(1), 118-129 (2004)

14. Landers, D.M., Han, M.W., Salazar, W., Petruzzello, S.J., Kubitz, K.A., et al.: Effects of learning on electroencephalographic and electrocardiographic patterns in novice archers. Int. J. Sport Psychol. 25, 313-330 (1994)

15. Slobounov, S., Ray, W., Cao, C., Chiang, H.: Modulation of cortical activity as a result of task-specific practice. Neurosci. Lett. 421(2), 126-131 (2007)

16. Kranczioch, C., Athanassiou, S., Shen, S., Gao, G., Sterr, A.: Short-term learning of a visually guided power-grip task is associated with dynamic changes in EEG oscillatory activity. Clin. Neurophysiol. 119(6), 1419-1430 (2008)

17. Caplan, J.B., Madsen, J.R., Schulze-Bonhage, A., Aschenbrenner-Scheibe, R., Newman, E.L., et al.: Human theta oscillations related to sensorimotor integration and spatial learning. J. Neurosci. 23(11), 4726-4736 (2003)

18. Yordanova, J., Falkenstein, M., Hohnsbein, J., Kolev, V.: Parallel systems of error processing in the brain. Neuroimage 22(2), 590-602 (2004)

19. Bell, M.A., Fox, N.A.: Crawling experience is related to changes in cortical organization during infancy: evidence from EEG coherence. Dev. Psychobiol. 29(7), 551-561 (1996)

20. Gentili, R.J., Bradberry, T.J., Hatfield, B.D., Contreras-Vidal, J.L.: Brain Biomarkers of Motor Adaptation Using Phase Synchronization. In: Proceedings of the IEEE International Conference of the Engineering in Medicine and Biology Society, Minneapolis, Minnesota, USA, September 2-6, vol. 1, pp. 5930-3 (2009)

21. Izzetoglu, M., Bunce, S.C., Izzetoglu, K., Onaral, B., et al.: Functional brain imaging using near-infrared technology. IEEE Eng. Med. Biol. Mag. 26(4), 8-46 (2007)

22. Leff, D.R., Orihuela-Espina, F., Atallah, L., Athanasiou, T., et al.: Functional prefrontal reorganization accompanies learning-associated refinements in surgery: a manifold embedding approach. Comput. Aided Surg. 13, 325-339 (2008) 
23. Gentili, R.J., Hadavi, C., Ayaz, H., Shewokis, P.A., Contreras-Vidal, J.L.: Hemodynamic Correlates of Visuomotor Adaptation by Functional Near Infrared Spectroscopy. In: IEEE EMBS Proceedings, Buenos Aires, Argentina, pp. 2918-2921 (2010)

24. Defense Advanced Research Projects Agency - Defense Science Office, http: / /www. darpa.mil/dso/thrusts/trainhu/accelerated/index.htm

25. Thompson, T., Steffert, T., Ros, T., Leach, J., Gruzelier, J.: EEG applications for sport and performance. Methods 45, 279-288 (2008)

26. Landers, D.M., Petruzzello, S.J., Salazar, W., Crews, D.L., Kubitz, K.A., Grannon, T.L., Han, M.: The influence of electrocortical biofeedback on performance in pre-elite archers. Medicine and Science in Sports and Exercise 23, 123-129 (1991)

27. Arns, M., Kleinnijenhuis, M., Fallahpour, K., Breteler, R.: Golf Performance Enhancement and Real-Life Neurofeedback Training Using Personalized Event-Locked EEG Profiles. J. Neurother. 11(4), 11-18 (2009)

28. Vernon, D., Egner, T., Cooper, N., Compton, T., Neilands, C., Sheri, A., Gruzelier, J.: The effect of training distinct neurofeedback protocols on aspects of cognitive performance. Int. J. Psychophysiol. 47(1), 75-85 (2003)

29. Egner, T., Gruzelier, J.H.: Ecological validity of neurofeedback: Modulation of slow wave EEG enhances musical performance. NeuroReport 14, 1221-1224 (2003)

30. Hoyt, R.W., Reifman, J., Coster, T.S., Buller, M.J.: Combat medical infomatics: Present and future. In: Proceedings of AMIA Symposium, pp. 335-339 (2002)

31. Oken, B.S., Salinsky, M.C., Elsas, S.M.: Vigilance, alertness, or sustained attention: physiological basis and measurement. Clin. Neurophys. 117(9), 1885-1901 (2006)

32. Miller, M.W., Rietschel, J., McDonald, C.G., Pangelinan, M., Bush, L., Hatfield, B.D.: EEG assessment of incremental changes in cognitive workload during an ecologically valid visuo-motor task. In: 40th SFN Meeting, San Diego, CA, USA, November 13-17 (2009)

33. Costanzo, M.E., Oh, H., Bulkley, B., Contreras-Vidal, J.L., Goodman, R., Haufler, A., Lo, L.C., et al.: Independent component analysis of brain processes under psychological stress during motor performance. In: 39th SFN Meeting, Chicago, IL, USA, October 17-21 (2009)

34. Makeig, S., Jung, T.P.: Changes in alertness are a principal component of variance in theEEG spectrum. NeuroReport 7, 213-216 (1995)

35. Miller, M.W., Rietschel, J., McDonald, C.G., Hatfield, B.D.: A Novel Approach to the Physiological Measurement of Mental Workload. International Journal of Psychophysiology (in press) 